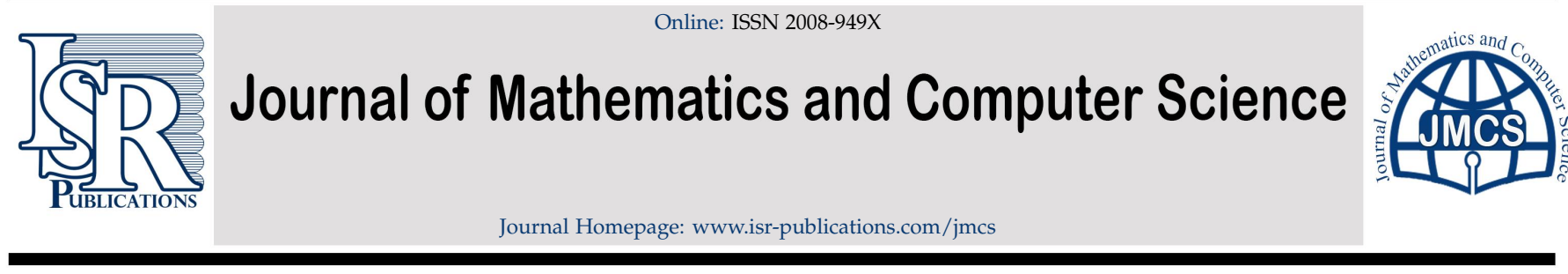

\title{
Hyers-Ulam-Rassias stability of abstract second-order linear dynamic equations on time scales
}

\author{
Maryam Alghamdia ${ }^{\text {, Alaa Aljehania }}{ }^{\mathrm{a}}$, Alaa E. Hamza ${ }^{\mathrm{b}, *}$ \\ ${ }^{a}$ Department of Mathematics, College of Science, University of Jeddah, Jeddah 21589, Saudi Arabia. \\ ${ }^{b}$ Department of Mathematics, Faculty of Science, Cairo University, Giza, Egypt.
}

\begin{abstract}
In this paper, we obtain new sufficient conditions for Hyers-Ulam and Hyers-Ulam-Rassias stability of abstract second-order linear dynamic equations on time scales. Also a new sufficient condition for the existence and uniqueness of solutions is established, via Banach's fixed point theorem. Finally, two illustrative examples are given to demonstrate the applicability of the theoretical results.
\end{abstract}

Keywords: Linear dynamic equations on time scales, Hyers-Ulam stability, Hyers-Ulam-Rassias stability.

2020 MSC: 34D20, 34N05, 39A30.

(C)2022 All rights reserved.

\section{Preliminaries and introduction}

In 1940, Ulam posed the following question related to the stability of functional equations: "What are conditions under which a linear mapping near an approximately linear mapping to exist?". See [33]. The case of approximately additive mappings was solved by Hyers [15] who proved that the Cauchy equation is stable in Banach spaces. This type of stability founded by Ulam and Hyers, famed for Hyers-Ulam stability. In 1978, Rassias [27] extended Hyers-Ulam stability concept and called it Hyers-Ulam-Rassias stability. Since the last decades many articles have appeared. See for example the results in $[3,5,16-18,22-26,28,29,32,36]$.

Wang et al. introduced Heyrs-Ulam stability of linear differential equations of first order [35]. In [4] Anderson, Gates and Heuer extend the work of $\mathrm{Li}$ and Shen $[19,20]$ to prove Hyers-Ulam stability of the scalar second-order linear nonhomogeneous dynamic equation on bounded time scales. Hamza and Yaseen [13] generalized these results for unbounded time scales.

In 2017, Shen [30] established the Ulam stability of the first-order linear dynamic equations and its adjoint equation on time scales by using the integrating factor method. See also [31]. Recently, there has been a great interest in Hyers-Ulam stability due to its applications in control theory, numerical analysis and mechanical problems etc. We refer the reader to take a look at the more recent articles $[1,2,6-8,11,12,21,34]$. Throughout the article, $\mathrm{X}$ is a Banach space endowed with a norm $\|$.$\| . We denote by$

$$
\mathrm{C}_{\mathrm{rd}}^{1}(\mathcal{J}, \mathrm{X})=\left\{\mathrm{f}: \mathcal{J} \rightarrow X \mid \mathrm{f}^{\Delta} \text { exists and rd-continuous }\right\},
$$

and

$$
\mathrm{C}_{\mathrm{rd}}^{2}(\mathcal{J}, X)=\left\{\mathrm{f}: \mathcal{J} \rightarrow X \mid f^{\Delta}, f^{\Delta^{2}} \text { exist and rd-continuous }\right\}
$$

\footnotetext{
${ }^{*}$ Corresponding author

Email addresses: maaalghamdi4@gmail.com (Maryam Alghamdi), a.a418@hotmail.com (Alaa Aljehani), hamzaaeg2003@yahoo.com (Alaa E. Hamza)
}

doi: $10.22436 /$ jmcs.024.02.02

Received: 2020-10-23 Revised: 2020-11-05 Accepted: 2020-11-16 
Here $\mathcal{J}:=[a, b] \cap \mathbb{T}$, where $\mathbb{T}$ is a time scale and $a, b \in \mathbb{T}$ with $a<b$. As usual for a bounded function $f$ from $\mathbb{T}$ to $X$, we denote by

$$
\|f\|_{\infty}=\sup _{t \in \mathbb{T}}\|f(t)\|
$$

In this paper, we extend the results of [14] concerning Hyers-Ulam and Hyers-Ulam-Rassias stability of the equation

$$
\psi^{\Delta^{2}}(\mathrm{t})+\mathrm{p}(\mathrm{t}) \psi(\mathrm{t})-\mathrm{f}(\mathrm{t})=0, \quad \mathrm{t} \in \mathcal{J}^{\mathrm{K}^{2}} .
$$

We aim to obtain new sufficient conditions for Hyers-Ulam and Hyers-Ulam-Rassias stability of the general secondorder linear dynamic equations on time scales of the form

$$
\psi^{\Delta^{2}}(t)+\mathcal{Q}(t) \psi^{\Delta}(t)+\mathcal{P}(t) \psi(t)-f(t)=0, \quad t \in \mathcal{J}^{k^{2}},
$$

where $\mathcal{P}, Q \in \mathrm{C}_{\mathrm{rd}}(\mathcal{J}, \mathbb{R})$ and $\mathrm{f} \in \mathrm{C}_{\mathrm{rd}}(\mathcal{J}, \mathrm{X})$, the space of all rd-continuous from $\mathcal{J}$ to $\mathrm{X}$. Our results depend basically on finding an integral equation which is equivalent to equation (1.1). An existence and uniqueness result of solutions is obtained, via Banach's fixed point theorem. The second result is obtained using the corresponding Riccati equation. For the terminology and notations used here, we refer the reader to the monographs of Bohner and Peterson [9] and [10]. Here, we only recall the basic definitions which they are essential in our investigations.

Definition 1.1. Let $\mathbb{T}$ be a time scale. The forward jump operator $\sigma: \mathbb{T} \rightarrow \mathbb{T}$ is defined by

$$
\sigma(t)=\inf \{s \in \mathbb{T}: s>t\},
$$

In this definition we put $\inf \emptyset=\sup \mathbb{T}$.

Definition 1.2. The graininess function $\mu: \mathbb{T} \rightarrow[0, \infty)$ is defined by

$$
\mu(t)=\sigma(t)-t
$$

\section{Definition 1.3.}

(1) For a function $f: \mathbb{T} \rightarrow X, f^{\sigma}(t)$ is understood to mean $f(\sigma(t))$.

(2) A function $f: \mathbb{T} \rightarrow X$ is said to be right-dense continuous or rd-continuous provided $f$ is continuous at all rightdense points in $\mathbb{T}$ and its left-sided limits exist (finite) at all left-dense points $t$ in $\mathbb{T}$. The set of all rd-continuous functions $f: \mathbb{T} \rightarrow X$ will be denoted by $C_{r d}(\mathbb{T}, X)$.

(3) Assume $f: \mathbb{T} \rightarrow X$, and let $t \in \mathbb{T}^{k}$. The delta-derivative of $f$ at $t$, denoted $f^{\Delta}(t)$, is defined to be the element of $X$ with the property that given any $\epsilon>0$, there is a neighborhood $\mathbf{U}$ of $t$ such that

$$
\left\|[f(\sigma(t))-f(s)]-f^{\Delta}(t)[\sigma(t)-s]\right\| \leqslant \epsilon|\sigma(t)-s|, \forall s \in \mathbf{U} .
$$

If $\mathrm{f}^{\Delta}(\mathrm{t})$ exists we say $\mathrm{f}$ is delta-differentiable at $t$, and we say $\mathrm{f}^{\Delta}: \mathbb{T}^{\mathrm{k}} \rightarrow X$ is the delta-derivative of $f$ on $\mathbb{T}^{\mathrm{k}}$. For the notion $\mathbb{T}^{k}$, see $[9$, page 2$]$. We denote by

$$
f^{\Delta \sigma}=\left(f^{\Delta}\right)^{\sigma} \text { and } f^{\sigma \Delta}=\left(f^{\sigma}\right)^{\Delta} .
$$

\section{Existence and uniqueness results}

In this section, we obtain new sufficient conditions for the existence and uniqueness of solutions of equation (1.1) instead of the regressiveness condition of the equation, that is $1-\mu(t) \mathcal{Q}(t)+\mu^{2}(t) \mathcal{P}(t) \neq 0$ for all $t \in \mathcal{J}^{k}$. See [9].

The next theorem indicates the equivalence between the existence of a solution of the scalar homogeneous second-order equation

$$
\psi^{\Delta^{2}}(\mathrm{t})+\mathcal{Q}(\mathrm{t}) \psi^{\Delta}(\mathrm{t})+\mathcal{P}(\mathrm{t}) \psi(\mathrm{t})=0, \quad \mathrm{t} \in \mathcal{J}^{\mathrm{K}^{2}},
$$

and the existence of a solution $z$ of the corresponding Riccati equation

$$
(1-\mu(t) z(t)) z^{\Delta}(t)+\mathcal{Q}(t) z(t)-z^{2}(t)-\mathcal{P}(t)=0, \quad t \in \mathcal{J}^{k^{2}} .
$$

See also [9]. 
Theorem 2.1. Assume that Riccati equation (2.2) has a solution $z$ that satisfies $1-\mu(t) z(t) \neq 0, t \in \mathcal{J}^{k}$. Then $e_{-z}(t, a)$ is a solution of equation (2.1). Conversely, if equation (2.1) has a solution $x$ with no zeros, then $z(t)=-\frac{x^{\Delta}(t)}{x(t)}$ is a solution of equation (2.2).

Proof. Let $z$ be a solution of (2.2) that satisfies $1-\mu(t) z(t) \neq 0, t \in \mathcal{J}^{k}$. The function $x(t)=e_{-z}(t, a)$ is a solution of equation (2.1). Indeed, for $t \in \mathcal{J}^{k^{2}}$, we have

$$
x^{\Delta}(\mathrm{t})=-z(\mathrm{t}) x(\mathrm{t})
$$

and

$$
\begin{aligned}
x^{\Delta \Delta}(\mathrm{t})+\mathcal{Q}(\mathrm{t}) \chi^{\Delta}(\mathrm{t})+\mathcal{P}(\mathrm{t}) x(\mathrm{t}) & =z^{2}(\mathrm{t}) x(\mathrm{t})-z^{\Delta}(\mathrm{t})(x(\mathrm{t})-z(\mathrm{t}) x(\mathrm{t}) \mu(\mathrm{t}))-\mathcal{Q}(\mathrm{t}) z(\mathrm{t}) x(\mathrm{t})+\mathcal{P}(\mathrm{t}) x(\mathrm{t}) \\
& =x(\mathrm{t})\left(z^{2}(\mathrm{t})-z^{\Delta}(\mathrm{t})(1-z(\mathrm{t}) \mu(\mathrm{t}))-\mathcal{Q}(\mathrm{t}) z(\mathrm{t})+\mathcal{P}(\mathrm{t})\right)=0 .
\end{aligned}
$$

Conversely, assume that $x$ is a scalar solution of equation (2.1) with no zeros. Define $z$ by

$$
z(t)=-\frac{x^{\Delta}(t)}{x(t)}, t \in \mathcal{J}^{k}
$$

Then

$$
z^{\Delta}(t)=-\frac{\chi(t) x^{\Delta \Delta}(t)-\left(x^{\Delta}(t)\right)^{2}}{x(t) x^{\sigma}(t)}
$$

Simple calculations show that

$$
z^{2}(t)-(1-z(t) \mu(t)) z^{\Delta}(t)-\mathcal{Q}(t) z(t)=\frac{x^{\Delta \Delta}(t)+\mathcal{Q}(t) x^{\Delta}(t)}{x(t)}=-\mathcal{P}(t), \quad t \in \mathcal{J}^{k^{2}}
$$

The following result establishes a new sufficient condition for the existence of a unique solution $x$ of the scalar equation (2.1) that satisfies the initial conditions $x^{\Delta^{i}}(a)=a_{i}, i=0,1$ for any $a_{0}, a_{1} \in \mathbb{R}$.

Theorem 2.2. Let $\mathbb{T}$ be a time scale such that the forward jump operator $\sigma$ is differentiable. Assume that Riccati equation (2.2) related to the scalar equation (2.1) has a solution $z$ that satisfies both $1-\mu(t) z(t) \neq 0$ and $1-\mu(t)\left(Q(t)-z^{\sigma}(t)\right) \neq 0, t \in \mathcal{J}^{k}$. Then equation (2.1) has a unique solution $x$ that satisfies the initial conditions $x^{\Delta^{i}}(a)=a_{i}, i=0,1$ for any $a_{0}, a_{1} \in \mathbb{R}$.

Proof. In view of Theorem 2.1, assume that $x(t)=e_{-z}(t, a)$ is a solution of equation (2.1). We look for another solution $y$ of the form

$$
y(t)=u(t) x(t)
$$

where $u$ is a scalar function which will be chosen such that $\{x, y\}$ is a fundamental set for equation (2.1). We have

$$
y^{\Delta}(t)=u(t) x^{\Delta}(t)+u^{\Delta}(t) x^{\sigma}(t)
$$

and consequently

$$
\begin{aligned}
y^{\Delta^{2}}(t)+\mathcal{Q}(t) y^{\Delta}(t)+\mathcal{P}(t) y(t)= & u(t) x^{\Delta^{2}}(t)+u^{\Delta}(t) x^{\Delta \sigma}(t)+u^{\Delta}(t) x^{\sigma \Delta}(t)+u^{\Delta^{2}}(t) x^{\sigma^{2}}(t) \\
& +\mathcal{Q}(t) u(t) x^{\Delta}(t)+\mathcal{Q}(t) u^{\Delta}(t) x^{\sigma}(t)+\mathcal{P}(t) u(t) x(t) \\
= & u^{\Delta^{2}}(t) x^{\sigma^{2}}(t)+u^{\Delta}(t) x^{\Delta \sigma}(t)+u^{\Delta}(t) x^{\sigma \Delta}(t)+\mathcal{Q}(t) u^{\Delta}(t) x^{\sigma}(t) .
\end{aligned}
$$

Thus $y$ is a solution of equation (2.1) if and only if $u$ satisfies the following equation

$$
u^{\Delta^{2}}(t) e_{-z}^{\sigma^{2}}(t, a)+u^{\Delta}(t)\left(e_{-z}^{\Delta \sigma}(t, a)+e_{-z}^{\sigma \Delta}(t, a)+\mathcal{Q}(t) e_{-z}^{\sigma}(t, a)\right)=0,
$$

which yields

$$
v^{\Delta}(\mathrm{t})+\mathrm{r}(\mathrm{t}) v(\mathrm{t})=0,
$$


where $r(t)=\frac{e_{-z}^{\Delta \sigma}(t, a)+e_{-z}^{\sigma \Delta}(t, a)+Q(t) e_{-z}^{\sigma}(t, a)}{e_{-z}^{\sigma^{2}}(t, a)}$ and $v(t)=u^{\Delta}(t)$. One can see that $1-\mu(t) r(t) \neq 0, t \in J^{k}$. Indeed, we have

$$
\begin{aligned}
e_{-z}^{\sigma^{2}}(t, a)-\mu(t)\left(e_{-z}^{\Delta \sigma}(t, a)+e_{-z}^{\sigma \Delta}(t, a)+Q(t) e_{-z}^{\sigma}(t, a)\right) & =e_{-z}^{\sigma}(t, a)-\mu(t)\left(e_{-z}^{\Delta \sigma}(t, a)+Q(t) e_{-z}^{\sigma}(t, a)\right) \\
& =(1-\mu(t) \mathcal{Q}(t)) e_{-z}^{\sigma}(t, a)-\mu(t) e_{-z}^{\Delta \sigma}(t, a) \\
& =(1-\mu(t) \mathcal{Q}(t)) e_{-z}^{\sigma}(t, a)+\mu(t) z^{\sigma}(t) e_{-z}^{\sigma}(t, a) \\
& =\left(1-\mu(t) \mathcal{Q}(t)+\mu(t) z^{\sigma}(t)\right) e_{-z}^{\sigma}(t, a) \\
& \neq 0 .
\end{aligned}
$$

Then $v(t)=e_{-r}(t, a)$ exists and is a solution of equation (2.3). Hence

$$
u(t)=\int_{a}^{t} e_{-r}(s, a) \Delta s
$$

The Wronskian of $x$ and $y=u x$ is given by

$$
\begin{aligned}
\mathcal{W}(x, y)(t) & =e_{-z}(t, a)\left(u(t) e_{-z}^{\Delta}(t, a)+u^{\Delta}(t) e_{-z}^{\sigma}(t, a)\right)-e_{-z}(t, a) u(t) e_{-z}^{\Delta}(t, a) \\
& =e_{-z}(t, a) e_{-z}^{\sigma}(t, a) u^{\Delta}(t)=e_{-z}(t, a) e_{-z}^{\sigma}(t, a) e_{-r}(t, a)
\end{aligned}
$$

It follows that the Wronskian $\mathcal{W}(x, y)(t) \neq 0, t \in \mathcal{J}$ and consequently $\{x, y\}$ is a fundamental set of equation (2.1). Then for any $a_{0}, a_{1} \in \mathbb{R}$, there exist $c_{1}, c_{2} \in \mathbb{R}$ such that the solution $\psi(t)=c_{1} x(t)+c_{2} y(t)$ of equation (2.1) satisfies the initial conditions $\psi^{\Delta^{i}}(a)=a_{i}, i=0,1$.

From now on, we assume $\mathcal{P} \in \mathrm{C}_{\mathrm{rd}}(\mathcal{J}, \mathbb{R})$ and $Q \in \mathrm{C}_{\mathrm{rd}}^{1}(\mathcal{J}, \mathbb{R})$. We need the following lemma in proving the rest of our results.

Lemma 2.3. $\psi$ is a solution of equation (1.1) if and only if $\psi$ satisfies the integral equation

$$
\begin{aligned}
\psi(t)= & a_{0}+a_{1}(t-a)+\mathcal{Q}(a) a_{0}(t-a)-\int_{a}^{t} \mathcal{Q}(s) \psi(s) \Delta s \\
& +\int_{a}^{t}[t-s-\mu(s)]\left[Q^{\Delta}(s) \psi(\sigma(s))-\mathcal{P}(s) \psi(s)+f(s)\right] \Delta s,
\end{aligned}
$$

for some constants $\mathrm{a}_{0}, \mathrm{a}_{1} \in \mathrm{X}$.

Proof. Assume $\psi$ satisfies the integral equation (2.4). We denote by

$$
M(t)=\mathcal{Q}(a) a_{0}(t-a)-\int_{a}^{t} \mathcal{Q}(s) \psi(s) \Delta s+\int_{a}^{t}[t-s-\mu(s)]\left[Q^{\Delta}(s) \psi(\sigma(s))-\mathcal{P}(s) \psi(s)+f(s)\right] \Delta s .
$$

By Theorem 1.117 in [9], we have

$$
M^{\Delta}(\mathrm{t})=\mathcal{Q}(\mathrm{a}) \mathrm{a}_{0}-\mathcal{Q}(\mathrm{t}) \psi(\mathrm{t})+\int_{\mathrm{a}}^{\mathrm{t}}\left[\mathrm{Q}^{\Delta}(\mathrm{s}) \psi(\sigma(\mathrm{s}))-\mathcal{P}(\mathrm{s}) \psi(\mathrm{s})+\mathrm{f}(\mathrm{s})\right] \Delta \mathrm{s},
$$

and

$$
M^{\Delta \Delta}(t)=-\mathcal{Q}(t) \psi^{\Delta}(t)-\mathcal{P}(t) \psi(t)+f(t) .
$$

Then

$$
\psi^{\Delta \Delta}(\mathrm{t})=M^{\Delta \Delta}(\mathrm{t})=-\mathcal{Q}(\mathrm{t}) \psi^{\Delta}(\mathrm{t})-\mathcal{P}(\mathrm{t}) \psi(\mathrm{t})+\mathrm{f}(\mathrm{t}) .
$$

To prove the second direction, assume that $\psi$ is a solution of equation (1.1). Integrating both sides of (1.1) two times, we get

$$
\psi(t)=a_{0}+a_{1}(t-a)-\int_{a}^{t} I(s) \Delta s
$$


where $\mathrm{I}(\mathrm{t})=\int_{\mathrm{a}}^{\mathrm{t}}\left[\mathrm{Q}(\mathrm{s}) \psi^{\Delta}(\mathrm{s})+\mathcal{P}(\mathrm{s}) \psi(\mathrm{s})-\mathrm{f}(\mathrm{s})\right] \Delta \mathrm{s}$. Consider the function

$$
L(t)=-\int_{a}^{t} I(s) \Delta s
$$

By taking $\Delta$-derivative of both sides of (2.5) and using (vi) of Theorem 1.77 in [9], we get

$$
\begin{aligned}
\mathrm{L}^{\Delta}(\mathrm{t})=-\mathrm{I}(\mathrm{t}) & =-\int_{\mathrm{a}}^{\mathrm{t}}\left[\mathcal{Q}(\mathrm{s}) \psi^{\Delta}(\mathrm{s})+\mathcal{P}(\mathrm{s}) \psi(\mathrm{s})-\mathrm{f}(\mathrm{s})\right] \Delta \mathrm{s} \\
& =-\int_{\mathrm{a}}^{\mathrm{t}} \mathcal{Q}(\mathrm{s}) \psi^{\Delta}(\mathrm{s}) \Delta \mathrm{s}-\int_{\mathrm{a}}^{\mathrm{t}}[\mathcal{P}(\mathrm{s}) \psi(\mathrm{s})-\mathrm{f}(\mathrm{s})] \Delta \mathrm{s} \\
& =-\mathcal{Q}(\mathrm{t}) \psi(\mathrm{t})+\mathcal{Q}(\mathrm{a}) \psi(\mathrm{a})+\int_{\mathrm{a}}^{\mathrm{t}} \mathrm{Q}^{\Delta}(\mathrm{s}) \psi(\sigma(s)) \Delta s-\int_{\mathrm{a}}^{\mathrm{t}}[\mathcal{P}(\mathrm{s}) \psi(\mathrm{s})-\mathrm{f}(\mathrm{s})] \Delta \mathrm{s} \\
& =M^{\Delta}(\mathrm{t}) .
\end{aligned}
$$

Consequently, by (iii) of Corollary 1.68 in [9], we obtain $M(t)=L(t)+C, t \in \mathcal{J}$. We have $C=M(a)-L(a)=0$. Therefore $\psi$ satisfies equation (2.4).

A sufficient condition for the existence of a unique solution of equation (1.1) can be stated in the following theorem.

Theorem 2.4. Assume

$$
\exists \alpha \in(0,1) \text { such that } \int_{a}^{t}\left[|\mathcal{Q}(s)|+(|t-s-\mu(s)|)\left(\left|\mathbb{Q}^{\Delta}(s)\right|+|\mathcal{P}(s)|\right)\right] \Delta s \leqslant \alpha, \mathrm{t} \in \mathcal{J} .
$$

Then for any $\mathrm{a}_{0}, \mathrm{a}_{1} \in \mathrm{X}$, equation (1.1) has a unique solution $\psi$ that satisfies the initial conditions

$$
\psi^{\Delta^{i}}(a)=a_{i}, i=0,1 .
$$

Proof. Fix $\mathrm{a}_{0}, \mathrm{a}_{1} \in X$. We prove that the operator $\mathrm{T}: \mathrm{C}_{\mathrm{rd}}(\mathrm{J}, \mathrm{X}) \rightarrow \mathrm{C}_{\mathrm{rd}}(\mathrm{J}, \mathrm{X})$ defined by

$$
\mathrm{T} \psi(\mathrm{t})=\mathrm{a}_{0}+\mathrm{a}_{1}(\mathrm{t}-\mathrm{a})+\mathcal{Q}(\mathrm{a}) \mathrm{a}_{0}(\mathrm{t}-\mathrm{a})-\int_{\mathrm{a}}^{\mathrm{t}} \mathcal{Q}(\mathrm{s}) \psi(\mathrm{s}) \Delta \mathrm{s}+\int_{\mathrm{a}}^{\mathrm{t}}[\mathrm{t}-\mathrm{s}-\mu(\mathrm{s})]\left[\mathrm{Q}^{\Delta}(\mathrm{s}) \psi(\sigma(\mathrm{s}))-\mathcal{P}(\mathrm{s}) \psi(\mathrm{s})+\mathrm{f}(\mathrm{s})\right] \Delta \mathrm{s}
$$

is a contraction. Indeed, for $\phi, \psi \in \mathrm{C}_{\mathrm{rd}}(\mathcal{J}, \mathrm{X})$, we have

$$
\|\mathrm{T} \psi(\mathrm{t})-\mathrm{T} \phi(\mathrm{t})\| \leqslant\left(\int_{\mathrm{a}}^{\mathrm{t}}\left[|\mathcal{Q}(\mathrm{s})|+(|\mathrm{t}-\mathrm{s}-\mu(\mathrm{s})|)\left(\left|\mathbb{Q}^{\Delta}(\mathrm{s})\right|+|\mathcal{P}(\mathrm{s})|\right)\right] \Delta \mathrm{s}\right)\|\psi-\phi\|_{\infty} \leqslant \alpha\|\psi-\phi\|_{\infty}, \mathrm{t} \in \mathcal{J} .
$$

This implies that $\|T \psi-T \phi\|_{\infty} \leqslant \alpha\|\psi-\phi\|_{\infty}$. Therefore $T$ has a unique fixed point $\psi$ which is the solution of the integral equation (2.4) satisfying the initial conditions.

As a direct consequence of Theorem 2.4, we obtain the following result.

Corollary 2.5. If the following condition

$$
(b-a)\|Q\|_{\infty}+(b-a)^{2}\left(\left\|Q^{\Delta}\right\|_{\infty}+\|\mathcal{P}\|_{\infty}\right)<1
$$

holds, then equation (1.1) has a unique solution $\psi$ that satisfies the initial conditions $\psi^{\Delta^{i}}(a)=a_{i}, i=0,1$ for any $a_{0}, a_{1} \in X$.

In Sections 3 and 4 , assume that $\mathcal{Q} \in \mathrm{C}_{r d}^{1}(\mathcal{J}, \mathbb{R}), \mathcal{P} \in \mathrm{C}_{\mathrm{rd}}(\mathcal{J}, \mathbb{R})$ and $f \in \mathrm{C}_{\mathrm{rd}}(\mathcal{J}, X)$. New sufficient conditions for Hyers-Ulam and Hyers-Ulam-Rassias stability of equation (1.1) are obtained. For the concepts of Hyers-Ulam and Hyers-Ulam-Rassias stability, see [4, 14]. 


\section{Hyers-Ulam stability results}

Definition 3.1. Equation (1.1) is said to have Hyers-Ulam stability if there is a constant $\mathrm{L}_{\mathrm{HU}}>0$ with the following property. For any $\epsilon>0$ and any $\psi \in \mathrm{C}_{\mathrm{rd}}^{2}(\mathcal{J}, X)$ satisfies

$$
\left\|\psi^{\Delta^{2}}(\mathrm{t})+\mathcal{Q}(\mathrm{t}) \psi^{\Delta}(\mathrm{t})+\mathcal{P}(\mathrm{t}) \psi(\mathrm{t})-\mathrm{f}(\mathrm{t})\right\| \leqslant \epsilon, \quad \mathrm{t} \in \mathcal{J}^{k^{2}},
$$

there exists a solution $\phi \in \mathrm{C}_{\mathrm{rd}}^{2}(\mathcal{J}, \mathrm{X})$ of equation (1.1) such that

$$
\|\psi(t)-\phi(t)\| \leqslant L_{H u s} \epsilon, \quad t \in \mathcal{J} .
$$

In this case $\mathrm{L}_{\mathrm{HU}}$ is called a Hyers-Ulam stability constant (HUs constant).

In the next theorem a new sufficient condition for Hyers-Ulam stability of equation (1.1) is established.

Theorem 3.2. Equation (1.1) has Hyers-Ulam stability with a HUs constant

$$
\mathrm{L}_{\mathrm{Hu}}:=\frac{(\mathrm{b}-\mathrm{a})^{2}}{1-\alpha}
$$

provided that condition (2.6) holds.

Proof. Let $\epsilon>0$ and $\psi \in \mathrm{C}_{\mathrm{rd}}^{2}(\mathcal{J}, \mathrm{X})$ satisfies inequality (3.1). Set

$$
h(t)=\psi^{\Delta^{2}}(t)+\mathcal{Q}(t) \psi^{\Delta}(t)+\mathcal{P}(t) \psi(t)-f(t) .
$$

Then $\psi$ solves the equation

$$
\psi^{\Delta^{2}}(t)+\mathcal{Q}(t) \psi^{\Delta}(t)+\mathcal{P}(t) \psi(t)=f(t)+h(t), \quad t \in \mathcal{J}^{k^{2}} .
$$

Let $a_{i}=\psi^{\Delta^{i}}(a), i=0,1$. By Lemma $2.3, \psi$ is the solution of the integral equation

$$
\begin{aligned}
\psi(t)= & a_{0}+a_{1}(t-a)+\mathcal{Q}(a) a_{0}(t-a)-\int_{a}^{t} \mathcal{Q}(s) \psi(s) \Delta s \\
& +\int_{a}^{t}[t-s-\mu(s)]\left[Q^{\Delta}(s) \psi(\sigma(s))-\mathcal{P}(s) \psi(s)+f(s)+h(s)\right] \Delta s
\end{aligned}
$$

Condition (2.6) ensures the existence of a unique solution $\phi$ of equation (1.1) with the initial conditions $\phi^{\Delta^{i}}(a)=a_{i}$, $i=0,1$. Equivalently, $\phi$ solves the integral equation

$$
\begin{aligned}
\phi(t)= & a_{0}+a_{1}(t-a)+\mathcal{Q}(a) a_{0}(t-a)-\int_{a}^{t} \mathcal{Q}(s) \phi(s) \Delta s \\
& +\int_{a}^{t}[t-s-\mu(s)]\left[\mathcal{Q}^{\Delta}(s) \phi(\sigma(s))-\mathcal{P}(s) \phi(s)+f(s)\right] \Delta s .
\end{aligned}
$$

From (3.6) and (3.7), we have

$$
\begin{aligned}
\|\psi(t)-\phi(t)\| & \leqslant \int_{a}^{t}|t-s-\mu(s)|\|h(s)\| \Delta s+\int_{a}^{t}\left[|\mathcal{Q}(s)|+(|t-s-\mu(s)|)\left(\left|Q^{\Delta}(s)\right|+|\mathcal{P}(s)|\right)\right] \Delta s\|\psi-\phi\|_{\infty} \\
& \leqslant(b-a)^{2} \epsilon+\alpha\|\psi-\phi\|_{\infty}, \mathrm{t} \in \mathcal{J}
\end{aligned}
$$

This implies that

$$
\|\psi-\phi\|_{\infty} \leqslant \frac{(b-a)^{2}}{1-\alpha} \epsilon .
$$

Consequently, $\phi$ satisfies (3.2). Therefore, equation (1.1) has Hyers-Ulam stability with a HUs constant L given by (3.3). 


\section{Hyers-Ulam-Rassias stability results}

Definition 4.1. Let $\mathcal{C}$ be a family of positive rd-continuous functions on $\mathcal{J}$. Equation (1.1) is said to have HyersUlam-Rassias stability of type $\mathcal{C}$ if there is a constant $\mathrm{L}_{\mathrm{HURs}}, \mathrm{C}>0$ with the following property. For any $\omega \in \mathcal{C}$ and any $\psi \in \mathrm{C}_{\mathrm{rd}}^{2}(\mathcal{J}, X)$ that satisfies

$$
\left\|\psi^{\Delta^{2}}(t)+\mathcal{Q}(t) \psi^{\Delta}(t)+\mathcal{P}(t) \psi(t)-f(t)\right\| \leqslant \omega(t), \quad t \in \mathcal{J}^{k^{2}},
$$

there exists a solution $\phi \in \mathrm{C}_{\mathrm{rd}}^{2}(\mathcal{J}, \mathrm{X})$ of equation (1.1) such that

$$
\|\psi(t)-\phi(t)\| \leqslant L_{\text {HuRs,e }} \omega(t), \quad t \in \mathcal{J} .
$$

In this case $\mathrm{L}_{\mathrm{HUR}, \mathrm{e}}$ is called a Hyers-Ulam-Rassias stability constant (HURs constant).

The following results are concerning with Hyers-Ulam-Rassias stability. Throughout the rest of the paper, we denote by

$$
\mathcal{M}_{\beta}^{p}=\left\{\omega \in C_{r d}(\mathcal{J}, \mathbb{R}): \omega \text { is positive, decreasing and } \int_{a}^{b} \omega^{p}(s) \Delta s \leqslant \beta \omega^{p}(b)\right\},
$$

where $p \geqslant 1$ and $\beta>0$.

Theorem 4.2. Assume that condition (2.6) holds. Then equation (1.1) has Hyers-Ulam-Rassias stability of type $\mathcal{M}_{\beta}^{1}$ with a HURs constant given by

$$
\mathrm{L}_{\mathrm{HUR}, \mathcal{M}_{\beta}^{1}}:=\frac{\beta(\mathrm{b}-\mathrm{a})}{1-\alpha}
$$

Proof. Let $\omega \in \mathcal{M}_{\beta}^{1}$ and $\psi \in \mathrm{C}_{\mathrm{rd}}^{2}(\mathcal{J}, X)$ satisfies inequality (4.1). Set $\mathrm{h}$ as in (3.4). Then $\psi$ is a solution of equation (3.5). By Lemma 2.3, it solves the integral equation (3.6), where

$$
a_{i}=\psi^{\Delta^{i}}(a), i=0,1
$$

By (2.6), there exists a unique solution $\phi$ of equation (1.1) with the initial conditions $\phi^{\Delta^{i}}(a)=a_{i}, i=0,1$. Equivalently, $\phi$ is the solution of the integral equation (3.7). Using equations (3.6) and (3.7), we conclude that

$$
\begin{aligned}
\|\psi(t)-\phi(t)\| & \leqslant \int_{a}^{t}|t-s-\mu(s)|\|h(s)\| \Delta s+\int_{a}^{t}\left(|\mathcal{L}(s)|(|t-s-\mu(s)|)\left(\left|\mathbb{Q}^{\Delta}(s)\right|+|\mathcal{P}(s)|\right)\right) \Delta s\|\psi-\phi\|_{\infty} \\
& \leqslant(b-a) \int_{a}^{t} \omega(s) \Delta s+\alpha\|\psi-\phi\|_{\infty} .
\end{aligned}
$$

Inequality (4.5) yields

$$
\|\psi-\phi\|_{\infty} \leqslant(b-a) \int_{a}^{b} \omega(s) \Delta s+\alpha\|\psi-\phi\|_{\infty} \leqslant \beta(b-a) \omega(b)+\alpha\|\psi-\phi\|_{\infty} \leqslant \beta(b-a) \omega(t)+\alpha\|\psi-\phi\|_{\infty}, t \in \mathcal{J} .
$$

It follows that

$$
\|\psi-\phi\|_{\infty} \leqslant \frac{\beta(b-a)}{1-\alpha} \omega(t), \quad t \in \mathcal{J}
$$

and consequently, $\phi$ satisfies (4.2). Therefore, equation (1.1) has Hyers-Ulam-Rassias stability of type $\mathcal{M}_{\beta}^{1}$ with a HURs constant given by (4.3).

Theorem 4.3. Assume that condition (2.6) holds. Let $\mathrm{p}>1$ and $\mathrm{q}=\frac{\mathrm{p}}{\mathrm{p}-1}$. Then equation (1.1) has Hyers-Ulam-Rassias stability of type $\mathcal{M}_{\beta}^{\mathrm{p}}$ with a HURs constant

$$
\mathrm{L}_{\text {HUR, } \mathcal{M}_{\beta}^{p}}:=\frac{\beta^{\frac{1}{p}}(b-a)^{\frac{q+1}{q}}}{1-\alpha}
$$


Proof. Let $\omega \in \mathcal{M}_{\beta}^{p}$ and $\psi \in C_{r d}^{2}(\mathcal{J}, X)$ satisfies (4.1). Define $h$ as in (3.4). Assume $\psi$ is given by (3.6), where $a_{i}$ is given by (4.4). Also assume that $\phi$ is the solution of (3.7). From (3.6) and (3.7), we obtain inequality (4.5). We apply Hölder inequality to get

$$
\begin{aligned}
\|\psi(t)-\phi(t)\| & \leqslant(b-a) \int_{a}^{b} \omega(s) \Delta s+\alpha\|\psi-\phi\|_{\infty} \\
& \leqslant(b-a)^{\frac{1}{q}}(b-a)\left(\int_{a}^{b} \omega^{p}(s) \Delta s\right)^{\frac{1}{p}}+\alpha\|\psi-\phi\|_{\infty} \leqslant \beta^{\frac{1}{p}}(b-a)^{\frac{q+1}{q}} \omega(b)+\alpha\|\psi-\phi\|_{\infty} .
\end{aligned}
$$

This implies

$$
\|\psi-\phi\|_{\infty} \leqslant \beta^{\frac{1}{p}}(b-a)^{\frac{q+1}{q}} \omega(b)+\alpha\|\psi-\phi\|_{\infty} \leqslant \beta^{\frac{1}{p}}(b-a)^{\frac{q+1}{q}} \omega(t)+\alpha\|\psi-\phi\|_{\infty}, \quad t \in \mathcal{J} .
$$

It follows that

$$
\|\psi-\phi\|_{\infty} \leqslant \frac{\beta^{\frac{1}{p}}(b-a)^{\frac{q+1}{q}}}{1-\alpha} \omega(t)
$$

Therefore, equation (1.1) has Hyers-Ulam-Rassias stability of type $\mathcal{M}_{\beta}^{p}$ with a HURs constant given by (4.6).

\section{Illustrative examples}

Example 5.1. Consider the equation

$$
\psi^{\Delta^{2}}(\mathrm{t})+e^{-1} \psi^{\Delta}(\mathrm{t})+r e^{\mathrm{t}-1} \psi(\mathrm{t})=\mathrm{f}(\mathrm{t}), \quad \mathrm{t} \in[0,1],
$$

where $r \in(0,1)$ and $f$ is continuous. Condition (2.6) holds with $\alpha=e^{-1}(1+r(e-1))<1$. Therefore, equation (5.1) has Hyers-Ulam stability with a constant

$$
\mathrm{L}_{\mathrm{HU}}=\frac{1}{1-\alpha}
$$

and has Hyers-Ulam-Rassias stability of type $\mathcal{M}_{\beta}^{p}$ with a constant

$$
\mathrm{L}_{\text {HURs, }, \mathcal{M}_{\beta}^{p}}=\frac{\beta^{\frac{1}{p}}}{1-\alpha}, p \geqslant 1 .
$$

Example 5.2. Let $n>1$ be a natural number and $\mathcal{J}=[1, n] \cap \mathbb{Z}$. Consider the equation

$$
\psi^{\Delta^{2}}(\mathrm{t})+\frac{d}{n-1} \psi^{\Delta}(t)+\frac{a(t)(1-d)}{n^{3}} \psi(t)=f(t), \quad t \in \mathcal{J}^{k^{2}},
$$

where $d \in(0,1), f \in C_{r d}(\mathcal{J}, \mathbb{R})$ and $a: \mathcal{J} \rightarrow[0,1]$. For $t \in \mathcal{J}$, we have

$$
\begin{aligned}
\int_{1}^{t}\left[|q(s)|+(|t-s-\mu(s)|)\left(\left|q^{\Delta}(s)\right|+|p(s)|\right)\right] \Delta s & \leqslant \int_{1}^{n} \frac{d}{n-1} \Delta s+n \int_{1}^{n} \frac{a(s)(1-d)}{n^{3}} \Delta s \\
& =d+\frac{(1-d)}{n^{2}} \int_{1}^{n} a(s) \Delta s \\
& =d+\frac{1-d}{n^{2}} \sum_{k=1}^{n-1} a(k) \\
& \leqslant d+\frac{(1-d)(n-1)}{n^{2}} \\
& <1 .
\end{aligned}
$$

Hence condition (2.6) holds with $\alpha=d+\frac{(1-d)(n-1)}{n^{2}}$. Therefore, equation (5.2) has Hyers-Ulam stability with a constant

$$
\mathrm{L}_{\text {HUs }}:=\frac{(\mathrm{n}-1)^{2}}{1-\alpha},
$$


and has Hyers-Ulam-Rassias stability of type $\mathcal{M}_{\beta}^{1}$ with a constant

$$
\mathrm{L}_{\text {HURs, M }}^{1}=\frac{\beta(\mathrm{n}-1)}{1-\alpha},
$$

and has Hyers-Ulam-Rassias stability of type $\mathcal{M}_{\beta}^{p}$ with a constant

$$
\mathrm{L}_{\text {HUR, }, \mathcal{M}_{\beta}^{p}}:=\frac{\beta^{\frac{1}{p}}(\mathrm{n}-1)^{\frac{\mathrm{q}+1}{\mathrm{q}}}}{1-\alpha} .
$$

\section{References}

[1] M. Alghamdi, M. Alharbi, M. Bohner, A. E. Hamza, Hyers-Ulam and Hyers-Ulam-Rassias stability of first-order nonlinear dynamic equations, Submitted. 1

[2] M. Alghamdi, A. Aljehani, M. Bohner, A. E. Hamza, Hyers-Ulam and Hyers-Ulam-Rassias stability of first-order linear dynamic equations, Publ. Inst. Math. (Beograd)(N.S.), (2020), To appear. 1

[3] C. Alsina, R. Ger,On some inequalities and stability results related to the exponential function, J. Inequal. Appl., 2 (1988), 373-380. 1

[4] D. R. Anderson, B. Gates, D. Heuer, Hyers-Ulam stability of second-order linear dynamic equations on time scales, Commun. Appl. Anal., 16 (2012), 281-292. 1, 2

[5] D. R. Anderson, M. Onitsuka, Hyers-Ulam stability of first-order homogeneous linear dynamic equations on time scales, Demonstr. Math., 51 (2018), 198-210. 1

[6] D. R. Anderson, M. Onitsuka, Hyers-Ulam stability for a discrete time scale with two step sizes, Appl. Math. Comput., $344 / 345$ (2019), 128-140. 1

[7] D. R. Anderson, M. Onitsuka, Best constant for Hyers-Ulam stability of second-order h-difference equations with constant coefficients, Results Math., 74 (2019), 16 pages.

[8] A. R. Baias, D. Popa, On Ulam stability of a linear difference equation in Banach spaces, Bull. Malays. Math. Sci. Soc., 43 (2020), 1357-1371. 1

[9] M. Bohner, A. Peterson, Dynamic equations on time scales, Birkhäuser, Boston, (2001). 1, 1.3, 2, 2, 2, 2

[10] M. Bohner, A. Peterson, Advances in dynamic equations on time scales, Birkhäuser, Boston, (2003). 1

[11] J. Brzdek, P. Wójcik, On approximate solutions of some difference equations, Bull. Aust. Math. Soc., 95 (2017), 476-481. 1

[12] C. Chen, M. Bohner, B. Jia, Ulam-Hyers stability of Caputo fractional difference equations, Math. Methods Appl. Sci., 42 (2019), 74617470. 1

[13] A. E. Hamza, N. A. Yaseen, Hyers-Ulam stability of abstract second-order linear dynamic equations on time scales, Int. J. Math. Anal., 8 (2014), 1421-1432.

[14] A. E. Hamza, M. Alghamdi, A. Aljehani, On Hyers-Ulam and Hyers-Ulam-Rassias stability of second-order linear dynamic equations on time scales, Adv. Dyn. Syst. Appl., 15 (2020), 99-111. 1, 2

[15] D. H. Hyers, On the stability of the linear functional equation, Proc. Nat. Acad. Sci. U.S.A., 27 (1941), $222-224.1$

[16] P. Gavruta, L. Gavruta, A new method for the generalized Hyers-Ulam-Rassias stability, Int. J. Nonlinear Anal. Appl., 1 (2010), 11-18. 1

[17] S.-M. Jung, Hyers-Ulam stability of linear differential equations of the first order (III), J. Math. Anal. Appl., 311 (2005), $139-146$.

[18] S.-M. Jung, Hyers-Ulam stability of linear differential equations of first order (II), Appl. Math. Lett., 19 (2006), 854-858. 1

[19] Y. Li, Y. Shen, Hyers-Ulam stability of nonhomogeneous linear differential equations of second order, Int. J. Math. Math. Sci., 2009 (2009), 7 pages. 1

[20] Y. Li, Y. Shen, Hyers-Ulam stability of linear differential equations of second order, Appl. Math. Lett., 23 (2010), 306-309. 1

[21] T. Li, G. Viglialoro, Analysis and explicit solvability of degenerate tensorial problems, Bound. Value Probl., 2018 (2018), 13 pages. 1

[22] T. Miura, On the Hyers-Ulam stability of a differentiable map, Sci. Math. Jpn., 55 (2002), 17-24. 1

[23] T. Miura, S. Miyajima, S.-E.Takahasi, A characterization of Hyers-Ulam stability of first order linear differential operators, J. Math. Anal. Appl., 286 (2003), 1136-146.

[24] T. Miura, S.-E. Takahasi, H. Choda, On the Hyers-Ulam stability of real continuous function valued differentiable map, Tokyo J. Math., 24 (2001), 467-476.

[25] D. Popa, Hyers-Ulam stability of the linear recurrence with constant coefficients, Adv. Difference Equ., 2005 (2005), $101-107$.

[26] D. Popa, I. Raşa, On the Hyers-Ulam stability of the linear differential equation, J. Math. Anal. Appl., 381 (2011), 530-537. 1

[27] T. M. Rassias, On the stability of the linear mapping in Banach spaces, Proc. Amer. Math. Soc., 72 (1978), 297-300. 1

[28] I. A. Rus, Ulam stability of ordinary differential equations, Stud. Univ. Babeş-Bolyai Math., 54 (2009), 125-133. 1

[29] I. A. Rus, Gronwall lemma approach to the Hyers-Ulam-Rassias stability of an integral equation, Springer, New York, 35 (2010), 147-152. 1

[30] Y. Shen, The Ulam stability of first order linear dynamic equations on time scales, Results Math., 72 (2017), 1881-1895. 1

[31] Y.-H. Shen, Y.-J. Li, Hyers-Ulam stability of first order nonhomogeneous linear dynamic equations on time scales, Commun. Math. Res., 35 (2019), 139-148. 1

[32] S.-E.Takahasi, T. Miura, S. Miyajima, On the Hyers-Ulam stability of the Banach space-valued differential equation $\mathrm{y}^{\prime}=\lambda y$, Bull. Korean Math. Soc., 39 (2002), 309-315. 1

[33] S. M. Ulam, A collection of mathematical problems, Interscience Publishers, New York-London, (1960). 1

[34] G. Viglialoro, J. Murcia, A singular elliptic problem related to the membrane equilibrium equations, Int. J. Comput. Math., 90 (2013), 2185-2196. 1

[35] G. Wang, M. Zhou, L. Sun, Hyers-Ulam stability of linear differential equations of first order, Appl. Math. Lett., 21 (2008), 1024-1028. 1

[36] A. Zada, S. O. Shah, S. Ismail, T. Li, Hyers-Ulam stability in terms of dichotomy of first order linear dynamic systems, Punjab Univ. J. Math., 49 (2017), 37-47. 1 